\title{
Perfil do registro sanitário de dispositivos intersomáticos na Agência Nacional De Vigilância Sanitária (Anvisa)
}

\author{
Profile of the sanitary registry of intersomatic devices in the National \\ Sanitary Surveillance Agency (Anvisa)
}

Recebido em: 16/07/2018 Aceito em: 26/02/2019
Luciana Ferreira Mattos COLLI; Anna Luisa Dantas MARTINS;

Lucio Mendes CABRAL

Programa de Pós-Graduação em Regulação e Vigilância Sanitária, Universidade Federal do Rio de Janeiro - UFRJ. Avenida Carlos Chagas Filho, 373. Cidade Universitária, CEP: 21941-590. Rio de Janeiro, RJ, Brasil. E-mail: colli.luciana@gmail.com

\section{ABSTRACT}

Health products are medical devices used in a variety of procedures. These products are subject to health surveillance, and to be marketed in Brazil they need to be registered at the National Health Surveillance Agency (Anvisa), which assesses the degree of risk and the impact of them on Public Health. The objective of the present study was to evaluate the profile of the sanitary registry of intersomatic devices or cages. There are currently 103 cages listed at Anvisa, with a total of 74 valid, 4 canceled, and 25 expired registrations. These records belong to both domestic and international manufacturers and are composed mostly of peek or titanium. Thus, the records showed that the profile of the holding companies varies both in nationality and location in the Country, as well as, in the raw material used in the manufacture of the devices.

Keywords: health products; intersomatic device; cage; sanitary registry

\section{RESUMO}

Produtos para saúde são dispositivos de uso médico utilizados em variados procedimentos e estão sujeitos à vigilância sanitária. A comercialização, no Brasil, requer o registro na Agência Nacional de Vigilância Sanitária (Anvisa), que avalia o grau de risco dos mesmos e o impacto na Saúde Pública. O objetivo do presente trabalho foi avaliar o perfil do registro sanitário de dispositivos intersomáticos ou cages. Atualmente existem 103 cages listados na Anvisa, perfazendo 74 registros válidos, 4 registros cancelados e 25 registros vencidos. Estes registros pertencem a empresas fabricantes nacionais e internacionais e sua composição prevalente é o peek ou titânio. Dessa forma, a análise dos registros apontou que o perfil das empresas detentoras do registro varia em nacionalidade, localização no país e na seleção da matéria prima utilizada na fabricação dos dispositivos.

Palavras chave: produtos para saúde; dispositivo intersomático; cage; registro sanitário. 


\section{INTRODUÇÃO}

Produtos para a saúde ou produtos médicos são legalmente definidos como equipamentos, aparelhos, materiais, artigos, sistemas de uso ou aplicação médica, odontológica ou laboratorial. São aqueles destinados a prevenção, diagnóstico, tratamento, reabilitação ou anticoncepção e que não utilizam meio farmacológico, imunológico ou metabólico na sua principal função em seres humanos. Nesse sentido, é uma classe de produtos sujeita à vigilância sanitária, com um número vasto de diferentes produtos e aplicações em áreas médicas específicas (1).

De modo a segmentar as categorias de produtos para saúde, foram elencadas as seguintes subdivisões: produto médico ativo; produto médico ativo para diagnóstico; produto médico ativo para terapia; produto médico de uso único; produto médico implantável; produto médico invasivo e produto médico invasivo cirurgicamente (1). A distinção aplicada às subdivisões possui como critério a indicação de uso e ainda se o dispositivo é descartável ou reutilizável.

A indústria de produtos para saúde é inovadora, proativa e com grande capacitação para suprir $90 \%$ das necessidades do mercado interno brasileiro. A indústria exporta para mais de 180 países, trazendo divisas e gerando cerca de 60 mil empregos no país. A produção nacional em 2017 gerou em torno de R \$ 8,8 bilhões, representando a manutenção de um mercado promissor. Nesse mesmo ano, o setor gerou 62.130 empregos (2).

No Brasil, o órgão responsável pela regulamentação dos produtos para saúde é a Agência Nacional de Vigilância Sanitária (Anvisa). A Agência tem o papel de proteger e promover a saúde da população, garantir a segurança sanitária de produtos e serviços e participar da construção e acesso. Uma das ações realizadas pela Anvisa para garantir a segurança da saúde da população é o procedimento de registro de produtos para saúde. Os produtos são avaliados quanto a sua segurança, eficácia e qualidade antes de serem comercializados $(3-6)$.

As Boas Práticas de Fabricação de Produtos para Saúde são descritas na - Resolução de Di- retoria Colegiada (RDC) $\mathrm{n}^{\circ} 16$, de 28 de março de 2013 (6) e deve ser seguida por fabricantes, importadores e distribuidores. Toda empresa que deseja registrar seu produto na Anvisa deve apresentar o Certificado de Boas Práticas de Fabricação (CBPF), conforme determina a resolução (RE) no 25, de 21 de maio de 2009 (7), entre outros documentos.

O registro de produtos para saúde na Anvisa é regulamentado por resoluções específicas, de acordo com a natureza do produto médico. Entretanto, há uma estrutura de normas adicionais que tratam do assunto, que são: $\mathrm{RDC} \mathrm{n}^{\circ}$ 185, de 22 de outubro de 2001(1), que dispõe sobre o registro, alteração, revalidação e cancelamento do registro de produtos médicos; RDC $\mathrm{n}^{\mathrm{o}} 40$, de 26 de agosto de 2015 (8), que define os requisitos do cadastro de produtos médicos; RDC n ${ }^{\circ}$ 56, de 06 de abril de 2001(9), que define os requisitos essenciais de segurança e eficácia; RDC 16 de 28 de março de 2013 (6), que trata dos requisitos essenciais de segurança e eficácia; e a Instrução Normativa (IN) $n^{0} 08$ de 26 de dezembro de 2013 (10), que estabelece a abrangência da aplicação dos dispositivos do Regulamento Técnico de Boas Práticas de Fabricação de Produtos Médicos e Produtos para Diagnóstico de Uso in vitro para empresas que realizam as atividades de importação, distribuição e armazenamento e dá outras providências.

A empresa detentora do registro de um produto para saúde deve ser uma empresa legalmente habilitada, com Autorização de Funcionamento de Empresas (AFE) para fabricação ou importação de produtos para saúde. De acordo com a RDC 185/01 (1), o distribuidor que solicitar registro sanitário de um produto médico se equipara ao importador. Sendo fabricante, aquela empresa que projeta, fabrica, rotula, embala ou esteriliza um produto para saúde. Comparativamente, a atividade de importação de produto para saúde é caracterizada por encaminhar ao Brasil produtos para saúde fabricado em país estrangeiro $(1,6)$.

Para o registro sanitário na Anvisa, a empresa solicitante necessita peticionar o processo no sítio eletrônico da agência e encaminhar uma série de 
documentos determinados pela legislação, estes de cunho burocrático e técnico. Tais documentos são denominados também pela Agência como "dossiê de registro", e é a base para a equipe técnica atestar documentalmente a segurança e eficácia do candidato ao registro, deferindo ou indeferindo o processo de registro sanitário.

Os produtos para saúde estão sujeitos a cadastro ou registro, e o processo será de acordo com o grau de risco do produto, que são categorizados em: I, II, III e IV. O processo de enquadramento é determinado e discriminado por regras presentes na legislação brasileira para registro de produtos para saúde. Tais regras são baseadas em tempo de permanência do produto no paciente e o quanto ele é invasivo (1). Após a classificação e o enquadramento de risco do produto, a empresa solicitante poderá protocolar o pedido de cadastro ou registro.

A documentação técnica do registro é composta de diversos documentos, sendo que os de importância singular para a avaliação técnica saõ: instrução de uso, dizeres de rotulagem, carta de autorização do fabricante e certificado de livre comercialização. A instrução de uso é um dos documentos mais críticos do processo de registro de produtos para saúde. Deve ser detalhada e conter informações gráficas, desenhos, descrição da tecnologia de funcionamento do produto, indicar acessórios, caso haja, e especificações, precauções, material de composição e método de esterilização (1).

A RDC 185/01 se aplica a grande maioria de produtos e equipamentos médicos fabricados atualmente, excetuando-se os de diagnóstico in vitro, que possuem legislação específica. A legislação em vigor disciplina o registro de produtos de uso e aplicação médica, como por exemplo: próteses ortopédicas, stents, lentes corretivas, curativos, campo cirúrgico, entre outros. Entre os produtos de ampla aplicação em ortopedia estão os dispositivos intersomáticos ou cages, que devem ter registro na Anvisa.

Os dispositivos intersomáticos ou cages são espaçadores vertebrais (10) e classificam-se como produtos médicos implantáveis. Essa subdivisão da classificação de produtos para saúde pode ser definida como qualquer produto médico projetado para ser totalmente introduzido no corpo humano ou para substituir uma superfície epitelial ou ocular por meio de intervenção cirúrgica, e deverá permanecer no local após a intervenção. Também é considerado um produto médico implantável, qualquer produto médico destinado a ser parcialmente introduzido no corpo humano por meio de intervenção cirúrgica e permanecer após esta intervenção por longo prazo (1).

A tecnologia de fusão com cage foi originada de uma cirurgia feita por Bagby e alguns cirurgiões veterinários, e o método de compressão e distração foi o princípio básico para fusão intervertebral com cage (11). Apesar de ter sido desenvolvido para solucionar problemas na coluna cervical, o cage de fusão de fibra de carbono (12) e o cage de titânio (13) foram usados primeiramente em fusões interlombares e então, usados para tratar lesões degenerativas espinhais cervicais (14-17). Atualmente existem estudos avaliando cages de ácido lático, L-lactídeo biorreabsorvível (18) (19).

Segundo Hübner e cols (2011) os dispositivos intersomáticos foram desenvolvidos para minimizar a morbidade da área doadora do enxerto (20). Por meio deste dispositivo é possível restaurar o suporte biomecânico imediato, promover a osteointegração do enxerto que, utilizado em menor quantidade e na forma de osso esponjoso, tem a função de preencher o dispositivo. Existem cages de diversos materiais, porém os mais utilizados são os de titânio e os de PEEK (poli-éter-éter-cetona) $(20,21,22)$. O cage ideal deve ter alta taxa de fusão e prevenção de complicações como subsidência e perda de correção (23).

Sendo os dispositivos intersomáticos produtos para saúde invasivos e de uso por longo prazo, mas que não interferem na função cardíaca do paciente, e por não entrarem em contato direto com o Sistema Nervoso Central, são classificados com grau de risco elevado, ou seja, risco III (1).

O presente trabalho teve como objetivo realizar um levantamento dos registros de dispositivos intersomáticos ou cages no sítio eletrônico da Anvisa e suas particularidades. As informações disponíveis no site são de livre acesso. 


\section{MÉTODO}

Foi realizada uma pesquisa descritiva e retrospectiva sob a forma de levantamento bibliográfico de artigos, revistas, livros e dissertações utilizando os seguintes termos: produtos para saúde, dispositivo intersomático, cage, registro sanitário. As fontes pesquisadas foram: Science Direct, PubMed, Scielo, Google Acadêmicos, Anvisa, sítio eletrônico da ABIMO (Associação Brasileira da Indústria de Artigos e Equipamentos Médicos). O período dos artigos considerados foi de 1999 a 2018. O levantamento realizado no sítio eletrônico da Anvisa foi estruturado para caracterizar arcabouço sanitário atual e vigente que regulamenta a fabricação, importação e Boas Práticas de Fabricação de Produtos para Saúde.

$\mathrm{Na}$ pesquisa realizada, a análise dos artigos e da legislação vigente teve como busca encontrar referência de quais os materiais são mais empregados na constituição e fabricação de cages e, ainda, como uma empresa deve proceder em termos regulatórios para produzir produtos para saúde adequados e obter deferimento em seus processos de registro.

Foi realizada, de forma complementar, uma busca no site da Anvisa, na área relativa a produtos para saúde, disponível no sítio eletrônico: http:// www7.anvisa.gov.br/datavisa/Consulta Produto correlato/consulta correlato.asp. Foi utlizado o termo cage e dispositivo intersomático que se encontram registrados na Agência. Como os dados foram obtidos: nome comercial do produto; nome técnico, CNPJ; número do processo de registro na Anvisa; instrução de uso e modelo de rotulagem. Foram considerados os registros publicados até maio de 2017.

\section{RESULTADOS E DISCUSSÃO}

A pesquisa realizada por registros relacionados à cages permitiu encontrar no sítio eletrônico da Anvisa o total de 103 registros sanitários e foram contabilizados os dispositivos intersomáticos de diferentes fabricantes que a agência dispunha no período.

Dos 103 cages listados no período da pesquisa, apenas 74 registros estavam válidos, 25 vencidos e
4 registros estavam cancelados, o que mostrou que apenas 74 produtos poderiam ser comercializados e utilizados em procedimentos cirúrgicos, sendo os demais, por força da legislação sanitária brasileira, inadequados para exposição à venda (3), conforme apresentado na Figura 1

Registros cancelados: 4 unidades

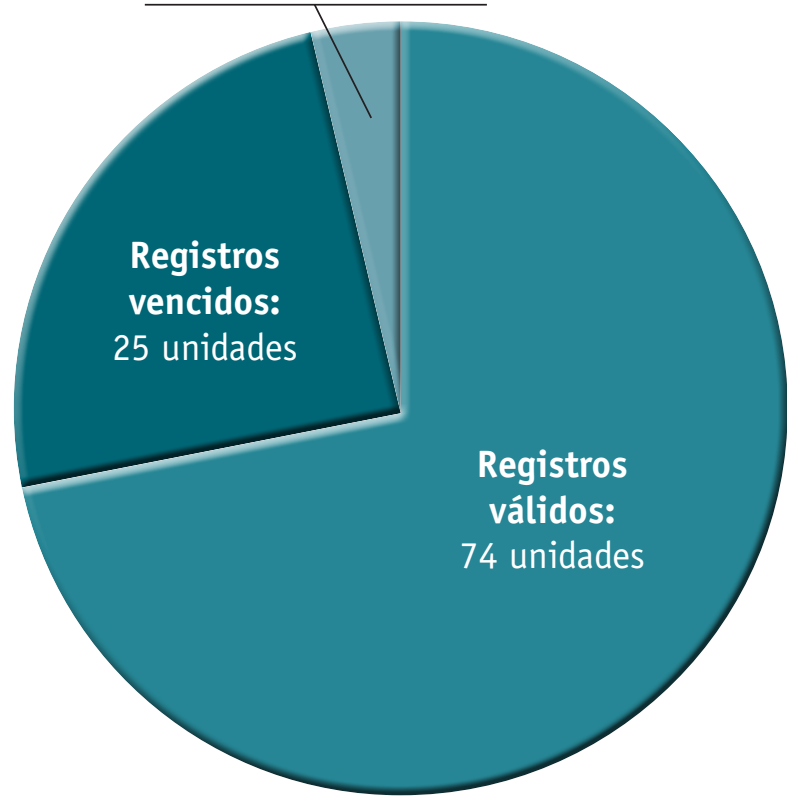

Figura 1. Distribuição dos registros de cage na Agência Nacional de Vgilância Sanitária (Anvisa), conforme listados em maio de 2017

Dentre o total de registros válidos, 39,8\% dos detentores de registro eram empresas fabricantes do produto em questão e não empresas que terceirizam a distribuição, que se caracterizam como depositários de registro, com atividade restrita de importação e comercialização. E $60,2 \%$ dos registros mapeados pertenciam a empresas com atividade específica de importação, isto é, representantes nomeados por um fabricante, usualmente instalados em outro País.

A quantidade de empresas importadoras era muito superior que aquelas fabricantes propriamente ditas, e seu quantitativo representa um número prevalente no mercado de produtos fabricados no exterior. Em contrapartida, 39,80\% eram fabricados em território nacional.

Em relação à distribuição geográfica dos detentores de registro, a maioria estava localizada na região Sudeste, mais precisamente em São Paulo (Figura 2). 


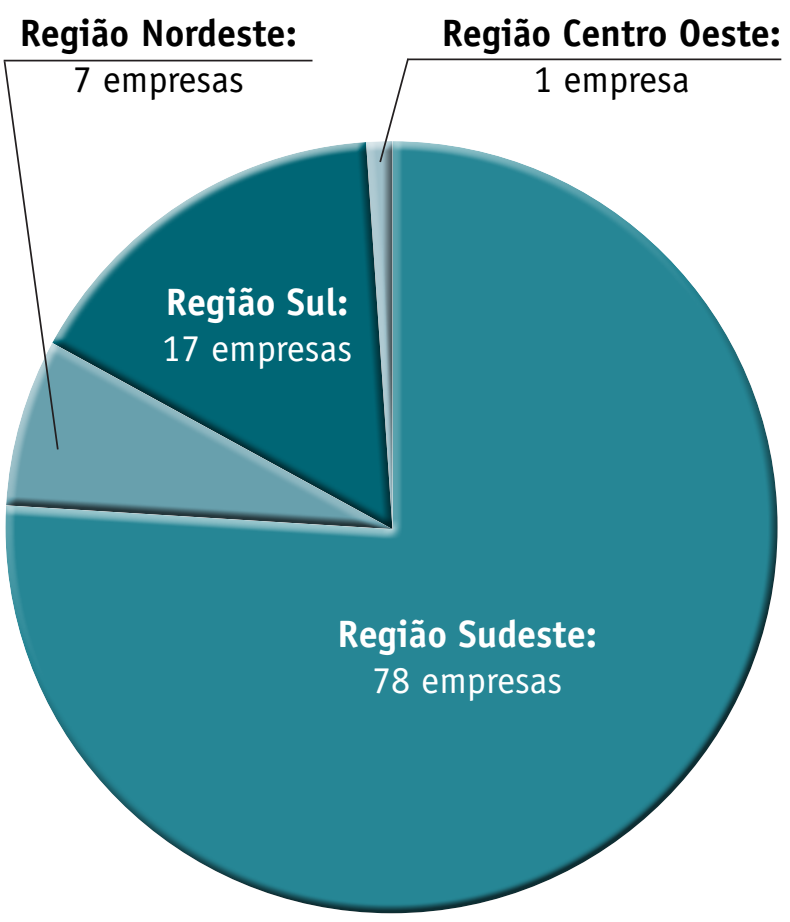

Figura 2. Distribuição geográfica dos detentores de registros de cage na Agência Nacional de Vigilância Sanitária (Anvisa), conforme listados em maio de 2017

Dentre os cages registrados, foi realizado um levantamento quanto ao país de origem dos fabricantes dos produtos. A maioria dos detentores era de origem nacional, seguidos dos Estados Unidos e França, sendo 35\% dos registros por detentores brasileiros, $23 \%$ dos registros pertencentes a americanos, $22 \%$ pertencem a franceses. Os países com menor número de detentor de registro de cage no Brasil eram a Espanha e Polônia (Figura 3).

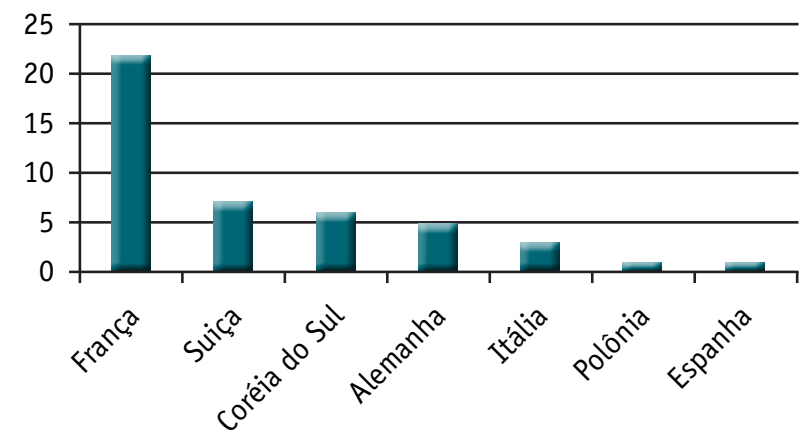

Figura 3. Origem dos fabricantes de cage conforme listados na Agência Nacional de Vigilância Sanitária (Anvisa), em maio de 2017

Em relação à disponibilidade de informações a respeito da composição e material utilizado na fabricação dos cages, os dados disponíveis na Anvisa permitiram determinar os componentes de fabricação e a natureza do material utilizado em $76,70 \%$ dos cages listados. Para $23,30 \%$ não havia detalhamento da composição. A maioria dos produtos tinha sua composição à base de PEEK (polyetheretherketona), um material plástico de elevado desempenho, produzido a partir da resina de poli-éter-éter-cetona, o que corrobora os estudos realizados por Hübner w cols (2011), Hwang e cols (2004) e Ray e cols (1997) (20 - 22). Os cages com fabricação a base de titânio representavam $16 \%$ dos produtos registrados e apenas 3\% utilizavam o material classificado como polimérico (Figura 4).

O levantamento buscou os componentes utilizados na fabricação de cages, e apesar de vários autores estudarem diferentes materiais, o PEEK ainda é o mais utilizado, seguido por titânio. Apesar das pesquisas evoluírem no uso de materiais biorreabsorvíveis, ainda não haviam sido peticionados e publicados, no período da pesquisa, registros de cages fabricados com ácido lático e L-lactídeo $(18,19)$, o que demonstra a morosidade dos processos de disponibilização de novas tecnologias aos pacientes e profissionais da saúde no Brasil, devido, em muitas vezes, aos processos regulatórios junto à Anvisa (24-26).

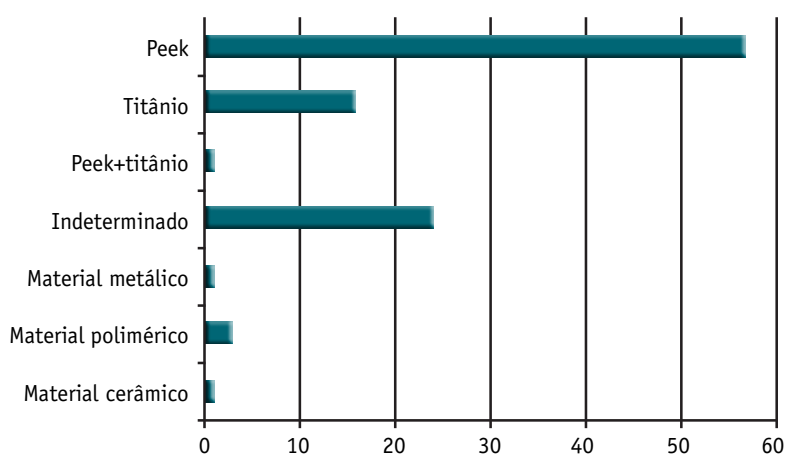

Figura 4. Material utilizado na composição dos cages, conforme listados na Agência Nacional de Vigilância Sanitária (Anvisa), em maio de 2017

Considerando que as informações estavam disponíveis na base de dados da Anvisa, é necessário que esta seja constantemente atualizada e alimentada. Contudo, tal fato não foi observado na "Pesquisa sobre Rotulagem e Instruções de Uso do Produto", disponibilizada pela Agência, cujos dados disponíveis eram a partir de junho de 2002. Foi possível observar que diversos dados após essa data também não estavam disponibilizados, tais como os modelos de rotulagem e instrução de uso 
de alguns produtos, o que dificultou a busca por informação.

É importante ressaltar que as informações disponíveis na base de dados da Anvisa dependem da correta classificação das empresas e de sua conferência pela Agência. Dessa forma, os produtos que não eram classificados como cage ou dispositivo intersomático em seu nome técnico e que não possuiam menção de "cage" ou "dispositivo intersomático" em seu nome comercial, não conseguiram ser capturados pela busca.

Surpreendentemente, quando comparado a outros produtos para saúde regulados pela Anvisa, foi verificado que o número de fabricantes de $c a$ ges nacionais é superior, seguido por fabricantes de produtos para saúde distribuídos em diversas outras nacionalidades. Tais ocorrências sugerem uma indústria nacional atuante e capacitada a atender a demanda interna de tratamento para patologias da coluna. A maioria dessas fábricas se localizava na região Sudeste, mais especificamente em São Paulo, devido ao maior desenvolvimento econômico e oferta de mão de obra especializada nessa região.

Por fim, este levantamento dos registros de $\mathrm{ca}$ ges no Brasil foi o início de um trabalho contínuo de assuntos regulatórios, traçando o panorama de registro por perfil de produto, que funciona como um auxiliar para profissionais que atuam na área e principalmente na elaboração dos dossiês de registro sanitário para os profissionais da área da saúde em geral. A disponibilização dessas informações possui grande relevância no processo de padronização destes produtos, de conduta terapêutica e de aquisição por hospitais e clínicas. A partir dessa avaliação, uma lista de cages registrados deverá ser periodicamente atualizada, considerando que a expiração e a concessão de novos registros são atividades dinâmicas e frequente dentre os objetivos de ação da Anvisa.

\section{CONCLUSÃO}

O registro de um produto para saúde é obrigatório e para a comercialização o produto precisa estar regular. As empresas importadoras ou fabricantes de produtos para saúde possuem estratégias de lançamento, inserção no mercado e comercialização dos mesmos. Instaladas em território nacional ou nomeando um importador é possível requerer registro sanitário.

O número de cages regulares mostrou que um número grande de empresas comercializava esses produtos. Como oposição, foi possível verificar aqueles com registro cancelado ou vencido, que apontam produtos cuja comercialização não se manteve.

O Brasil possui um número relevante de fabricantes e detentores do registro de cages instalados. Tal fato demonstra que há produção significativa deste produto em território nacional e aquecimento quanto ao desenvolvimento e fabricação de materiais médicos.

Com relação à matéria prima na composição dos cages, o PEEK (poli-éter-éter-cetona) era o componente amplamente empregado em sua composição, na sequência ficando o uso de titânio na composição destes dispositivos.

A importância da análise dos registros consiste em conhecer o perfil dos detentores, o comportamento ao longo do tempo e a classificação de risco de um produto para saúde, impactando na segurança e eficácia do tratamento.

\section{AGRADECIMENTOS}

Agradecemos a todos os envolvidos no processo de registro e ao pessoal do Atendimento ao Público da Anvisa 


\section{REFERÊNCIAS}

1. BRASIL. Aprova o Regulamento Técnico que trata do registro, alteração, revalidação e cancelamento do registro de produtos médicos na Agência Nacional de Vigilância Sanitária - ANVISA. Agência Nacional de Vigilância Sanitária. Resolução de Diretoria Colegiada (RDC) $\mathrm{n}^{\circ}$ 185, de 22 de outubro de 2001. Diário Oficial da União, n 201 de 06 nov. 2001. Seção 01. p. 54.

2. ABIMO. Dados Econômicos de Equipamentos Médicos, Odontológicos, Hospitalares e de Laboratórios no Brasil. Associação Brasileira da Indústria de Artigos e Equipamentos Médicos [Acesso em 28 dez. 2018]. Disponível em: https://abimo.org.br/dados-do-setor/dados-economi$\cos /$.

3. BRASIL. Dispõe sobre a vigilância sanitária a que ficam sujeitos os medicamentos, as drogas, os insumos farmacêuticos e correlatos, cosméticos, saneantes e outros produtos, e dá outras providências. Congresso Nacional. Lei $\mathrm{n}^{\circ}$ 6360, de 23 de setembro de 1976. Diário Oficial da União de 24 set. 1976.

4. BRASIL. Define o Sistema Nacional de Vigilância Sanitária, cria a Agência Nacional de Vigilância Sanitária, e dá outras providências. Congresso Nacional. Lei $n^{\circ}$ 9.782, de 26 de janeiro de 1999. Diário Oficial da União de 27 jan. 1999.

5. Costa EA, Aith F, Minhoto LD...[et al]. Vigilância sanitária: temas para debate. Salvador: EDUFBA, 2009. p.240.

6. BRASIL. Aprova o Regulamento Técnico de Boas Práticas de Fabricação de Produtos Médicos e Produtos para Diagnóstico de Uso In Vitro e dá outras providências. Agência Nacional de Vigilância Sanitária. Resolução de Diretoria Colegiada (RDC) $n^{\circ} 16$, de 28 de março de 2013. Diário Oficial da União, ${ }^{\circ} 61$ de 01 abr. 2013. Seção 1. p.75.

7. BRASIL. Exige o Certificado de Boas Práticas de Fabricação para o registro de Produtos para Saúde. Agência Nacional de Vigilância Sanitária. Resolução de Diretoria Colegiada (RDC) $n^{\circ} 25$, de 21 de maio de 2009. Diário Oficial da União, n 96 de 22 mai. 2009. Seção 1. p.48.

8. BRASIL. Define os requisitos do cadastro de produtos médicos. Agência Nacional de Vigilância Sanitária. Resolução de Diretoria Colegiada (RDC) n 40, de 26 de agosto de 2015. Diário Oficial da União, n 164 de 27 ago. 2015. Seção 1. p.47.

9. BRASIL. Os produtos para saúde devem atender aos requisitos essenciais de segurança e eficácia. Agência Nacional de Vigilância Sanitária. Resolução de Diretoria Colegiada (RDC) n 56, de 06 de abril de 2001. Diário
Oficial da União, n 70 de 10 abr. 2001. Seção 1. p.28.

10. BRASIL. Estabelece a abrangência da aplicação dos dispositivos do Regulamento Técnico de Boas Práticas de Fabricação de Produtos Médicos e Produtos para Diagnóstico de uso in vitro. Agência Nacional de Vigilância Sanitária. Instrução Normativa (IN) n ${ }^{\circ} 08$, de 26 de dezembro de 2013. Diário Oficial da União, n 252 de 30 dez. 2013. Seção 1. p.754.

11. Bagby GW. Arthrodesis by the distraction-compression method using a stainless steel implant. Am J Orthop. 1988; 11(6): 931-934. DOI: 10.3928/0147-744719880601-13.

12. Brantigan JW, Steffee AD. A carbon fiber implant to aid interbody lumbar fusion. Two-year clinical results in the first 26 patients. Eur. Spine J. 1993;18(14): 2106-2107. DOI: $10.1186 / \mathrm{s} 12891-016-1234-1$.

13. Grob D, Sylvia DAF. Titanium mesh cages (TMC) in spine surgery. Eur Spine J. 2005; 14:211-221. DOI: 10.1007/s00586-004-0748-7.

14. Hacker RJ, Cauthen JC, Gilbert TJ, Griffith SL. A prospective randomized multicenter clinical evaluation of an anterior cervical fusion cage. Eur Spine J. 2000; 25(20): 2646-2655.

15. Profeta G, Falco R, Ianniciello G, Profeta L, Cigliano A, Raja AI. Preliminary experience with anterior cervical microdiscectomy and interbody titanium cage fusion (Novus CT-Ti) in patients with cervical disc disease. Surg Neurol. 2000;53(5): 417-426. DOI: 10.1016/ S0090-3019(00)00207-X.

16. Machado FG, Ishi MM. Clinical outcomes with non-fusion stabilization in low back pain. Coluna/Columna. 2011;10(4): 329-331. DOI: 10.1590/S180818512011000400017 .

17. Amaral R, Ferreira R, Marchi L, Jensen R, NogueiraNeto J, \& Pimenta L. Artrodese lombar intersomática anterior por via única-Complicações e resultados perioperatórios. Rev. Bras. Ortop. 2017;52(5): 569-574. DOI: 10.1016/j.rbo.2016.09.006

18. Simões MS. Desenvolvimento e estudo in vitro de implante biorreabsorvível em poli (L-lactídeo) (PLLA) para artrodese de coluna lombar. [Dissertação]. Rio Grande do Sul: Pós-Graduação em Engenharia e Tecnologia de Materiais, Pontifícia Universidade Católica. 2007.

19. Goes Filho PRS. Confecção e avaliação mecânica de implantes ortopédicos produzidos em poli (L-ácido lático) 
(PLLA) por impressoras 3D. [Dissertação]. Pernambuco: Pós-Graduação em Biociência Animal, Universidade Federal Rural de Pernambuco. 2016.

20. Hübner AR, Mendes, MR Queruz, JCF, Dambrós, JM. Avaliação do tratamento da discopatia degenerativa cervical pela artrodese via anterior utilizando placas associadas a cages ou cages em peek isoladamente. Coluna/ Columna. 2011;10(4): 300-304. DOI: 10.1590/S180818512011000400010 .

21. Hwang SL, Lin CL, Lieu AS, Lee KS, Kuo TH, Hwang YF, et al. Three-level and four-level anterior cervical discectomies and titanium cage-augmented fusion with and without plate fixation. Spine. 2004;1(2): 160-167. DOI: 10.3171/spi.2004.1.2.0160.

22. Ray CD. Threaded titanium cages for lumbar interbody fusions. Spine. 1997; 22(6): 667-679.
23. Schimmel JJ, Poeschmann MS, Horsting PP, Schönfeld DH, van Limbeek, J, \& Pavlov, PW. PEEK cages in lumbar fusion: mid-term clinical outcome and radiologic fusion. Clin Spine Surg. 2016; 29(5):E252-E258. DOI: 10.1097/BSD.0b013e31826eaf74.

24. Novaes HMD. Da produção à avaliação de tecnologias dos sistemas de saúde: desafios do século XXI. Rev de Saúde Publ. 2006; 40: 133-140. DOI: 10.1590/S003489102006000400018.

25. Trindade E. A incorporação de novas tecnologias nos serviços de saúde: o desafio da análise dos fatores em jogo. Cad. de Saúde Pública. 2008;24: 951-964. DOI: 10.1590/ S0102-311X2008000500002.

26. Buvinich DPR. Ouvidoria: uma análise de sua utilização como ferramenta de gestão no âmbito da Agência Nacional de Vigilância Sanitária. [Dissertação]. Bahia: Instituto de Saúde Coletiva, Universidade. 